\title{
Grating acuity along the vertical meridian as a function of grating orientation
}

\author{
FREDERICK L. KITTERLE, RUSSELL S. KAYE, and JOHN SAMUELS \\ University of Toledo, Toledo, Ohio 43606
}

\begin{abstract}
Visual acuity was measured for horizontal, vertical, and oblique grating target orientations in the fovea and at various retinal eccentricities along the vertical meridian. Acuity was higher in the fovea, higher for vertical and horizontal target orientations, and higher in the superior retinal regions. The difference between acuities for vertical and oblique targets was greater in the superior retinal regions. It was concluded that the oblique effect is channel-size dependent.
\end{abstract}

In studies of visual acuity, resolution of foveally viewed sinusoidal grating targets tends to be better for horizontally or vertically oriented targets than for obliquely oriented gratings (Campbell, Kulikowski, \& Levinson, 1966; Higgins \& Stultz, 1950; Taylor, 1963). Appelle (1972) has named this phenomenon the oblique effect. Berkley, Kitterle, and Watkins (1975) measured acuity for horizontal, vertical, and oblique grating targets at several eccentricities along the horizontal meridian of the temporal retina. They found with foveal viewing that acuity was poorer for obliquely oriented targets than for the other orientations. However, with peripheral viewing, the differences between vertical and oblique acuity diminished and was found to be virtually absent at 8-18 deg. Berkely et al. (1975) concluded that the oblique effect depends upon the size of sized tuned channels. Their conclusion is supported by electrophysiological evidence (e.g., Watkins \& Berkley, 1974).

Millodot and Lamont (1974) measured peripheral visual acuity along the vertical meridian with Landolt rings and found acuity to be better in the superior retina than in the inferior retina at eccentricities beyond $5 \mathrm{deg}$.

While the exact neural mechanism underlying visual acuity is unknown, it has been suggested that visual acuity may in part be due to the size of retinal receptive fields (Rolls \& Cowey, 1970). Acuity is better in the fovea and receptive field size is smaller there than in the periphery, where acuity is poorer and the size of the receptive fields is considerably larger. Another conclusion that can be drawn from these results is that beyond $5 \mathrm{deg}$, receptive fields in the upper retinal regions are somewhat smaller than those in the lower visual field. If the visual channels or neural elements in the upper and lower visual fields are differentially sized tuned, then the oblique effect should be larger in the upper retinal region if the oblique effect is channel-size dependent, as hypothesized by Berkley et al. (1975).

\section{METHOD}

\section{Subjects}

Two male subjects, J.S. (19 years) and R.S.K. (25 years), participated in this experiment. Both were judged to have normal or corrected-to-normal vision.

\section{Apparatus}

The stimuli consisted of sinusoidal gratings that were generated on the face of an oscilloscope. Changes in the orientation of the gratings were accomplished by rotating the oscilloscope on a specially designed stand located behind a large rectangular screen that was at a viewing distance of $109 \mathrm{~cm}$ from the observer. At this distance, the screen subtended visual angles of $32 \times 26 \mathrm{deg}$. The gratings were viewed through a 2-deg circular aperture in the screen. The background was illuminated with a projector whose intensity and color were adjusted to match the oscilloscope screen in color and mean luminance. The luminance of the background was $6.5 \mathrm{~cd} / \mathrm{m}^{2}$, and the contrast of the gratings was $60 \%$. Small black dots $(10 \mathrm{~min})$ placed at various distances from the target formed the fixation points for viewing the target at various eccentricities, which ranged from $0 \mathrm{deg}$ (fovea) to 14 deg in 2-deg steps.

The display was viewed with the right eye; the left was patched during the experiment. A head holder was used to provide stable positioning of the head.

\section{Procedure}

At the beginning of the experimental session, the observer adapted to the mean luminance for $7 \mathrm{~min}$. After this, the grating was flashed for $200 \mathrm{msec}$, followed again by the uniform field for $800 \mathrm{msec}$. Grating acuity was determined by the experimenter's increasing or decreasing the spatial frequency of the grating until the subject signaled that he could just perceive it. Thresholds were determined at the various retinal eccentricities in a random manner. Four determinations were made for each condition, and the entire experiment was replicated four times on 4 successive days.

\section{RESULTS AND DISCUSSION}

In Figure 1A the results for R.S.K. are presented, and in Figure 1B the results for J.S. are shown. In Figure 1, threshold spatial frequency (left ordinate) or visual acuity (right ordinate) is plotted as a function of retinal eccentricity. The standard deviations for all points were rather small (.03-.05 min for central viewing and $.04-08 \mathrm{~min}$ for $12-$ to $14-\mathrm{deg}$ viewing angles). They are not shown in Figure 1 because a line of \pm 1 SD is smaller than the diameter of the symbols used to indicate the data points.

In general, these results parallel those of Millodot 

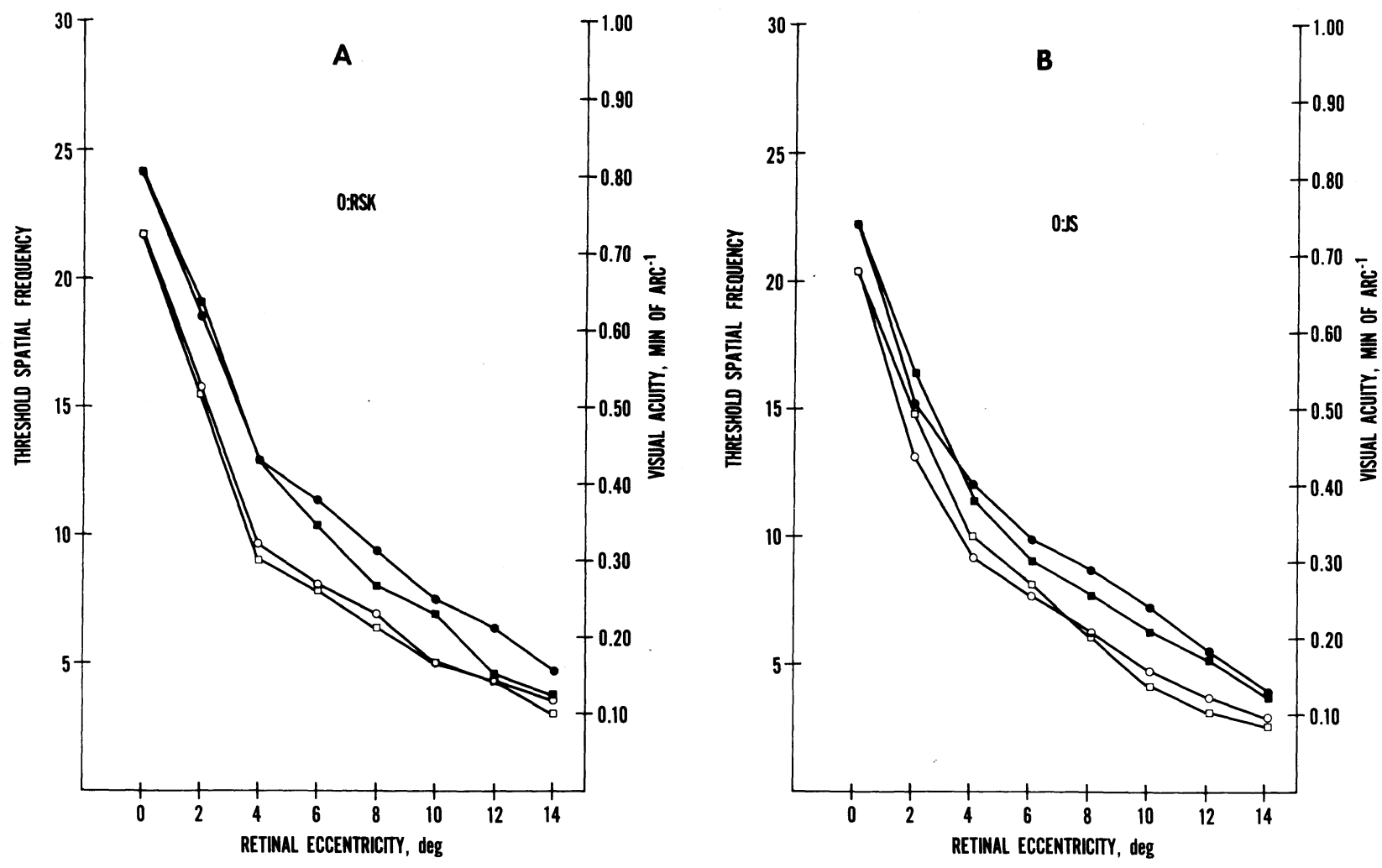

Figure 1. Visual acuity (threshold spatial frequency) plotted as a function of retinal eccentricity for vertical (filled circles) and oblique gratings (open circles) presented to the superior retinal regions and for vertical (filled squares) and oblique (open squares) gratings presented to the inferior retinal region. (A) Results for R.S.K. are on the left. (B) Results for J.S. are on the right.

and Lamont (1974). These figures show that visual acuity drops rather abruptly with off-axis viewing for both retinal regions. It is quite apparent that beyond 4-6 deg, acuity is significantly higher for the superior retinal regions for both the vertically and obliquely oriented gratings.

The oblique effect, which can be seen as a difference in acuity between the two orientations, is approximately the same for eccentricities less than 4 deg in the superior and inferior retina. However, beyond $4 \mathrm{deg}$, the magnitude of the oblique effect is significantly larger on the superior retina.

In summary, these results indicate that significant differences exist in visual acuity between the upper and lower retinal regions beyond 4-6 deg. At these eccentricities, the superior retinal regions have significantly higher resolution acuities than the inferior regions and also manifest a significantly greater oblique effect. Thus, the results of this experiment are consistent with the hypothesis that higher visual acuities are associated with smaller sized neural channels and that the oblique effect is channel-size dependent, as suggested by Berkley et al. (1975).

\section{REFERENCES}

Appelle, S. Perception and discrimination as a function of stimulus orientation: The "oblique effect" in man and animals. Psychological Bulletin, 1972, 78, 266-278.

Berkley, M. A., Kitterle, F. L., \& Watkins, D. W. Grating visibility as a function of orientation and retinal eccentricity. Vision Research, 1975, 15, 239-244.

Campbell, F. W., Kulikowski, J. J., \& Levinson, J. The effect of orientation on the visual resolution of gratings. Journal of Physiology, 1966, 187, 427-436.

Higgins, G. C., \& Stultz, K. Visual acuity as measured with various orientations of a parallel test object. Journal of the Optical Society of America, 1950, 38, 756-758.

Millodot, M., \& Lamont, A. Peripheral visual acuity in the vertical plane. Vision Research, 1974, 14, 1497-1498.

Rolls, E. T., \& CowEY, A. Topography of the retina and striate cortex and its relationship to visual acuity in rhesus monkeys and squirrel monkeys. Experimental Brain Research, 1970, 10, 298-310.

TAYLOR, M. M. Visual discrimination and orientation. Journal of the Optical Society of America, 1963, 53, 763-765.

Watkins, D., \& BERKLEY, M. A. The orientation selectivity of single neurons in cat striate cortex. Experimental Brain Research, $1974,19,433-446$.

(Received for publication October 30, 1980.) 